\title{
CIGRE Session 2018
}

\section{G. Christiner}

Online publiziert am 4. Dezember 2018

(c) Springer-Verlag GmbH Austria, ein Teil von Springer Nature 2018
CrossMark

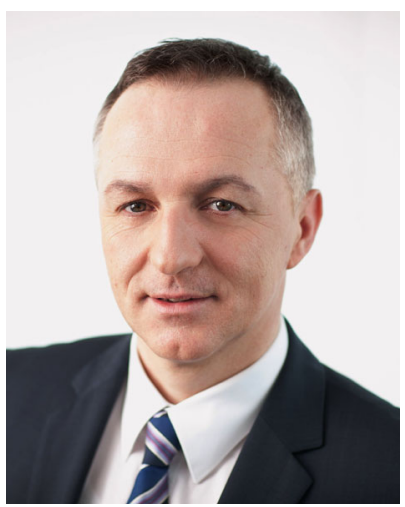

VDir. Dipl.-Ing. Mag. (FH) Gerhard Christiner
Die 47. CIGRE Session fand vom 26.-31. August 2018 in Paris statt. Als weltweit bedeutendste Plattform ihrer Art bietet die CIGRE Session alle zwei Jahre Vertretern der Energiewirtschaft und Industrie, ElektrotechnikExperten und Wissenschaftlern die Gelegenheit zum Erfahrungs- und Wissensaustausch.

Bei der diesjährigen Session kamen rund 3.800 Delegierte aus über 100 Ländern zur Diskussion aktueller Entwicklungen im Bereich der Energiewirtschaft zusammen. Auch das Interesse an der parallel zur Session stattfindenden Ausstellung war mit über 10.000 Besuchern enorm.

Die Schwerpunktthemen 2018 waren unter anderem:

- Digitalisierung/Big Data

- Dezentrale Energiesysteme und Speicher

- System Environmental Performance

- Alterungsprozesse
- Versorgungssicherheit

- DC-Systeme

Seit der letzten Session hat die CIGRE einen umfassenden Adaptierungsprozess durchlaufen, um für die Herausforderungen der Zukunft eine optimale Plattform zu bieten. Dies inkludiert auch neue Themenfelder im Bereich der Stromverteilung und Verbraucher, die im Rahmen der fortschreitenden Digitalisierung stärker mit Erzeugung und Übertragung interagieren. Die Digitalisierung ist auch stark im Energiemarkt mit Themen wie virtuelle Transaktionen vertreten. Dieser Erneuerungsprozess wurde mit der Überarbeitung des CIGRE-Logos und des Webauftrittes (https://www.cigre.org) im Rahmen der Session 2018 abgeschlossen.

In den einzelnen Arbeitsgruppen und Workshops ging es dann unter anderem um die Grundsatzfrage, welche Auswirkungen sich auf die einzelnen Arbeitsbereiche aus den aktuellen Systementwicklungen ableiten lassen und wo die Lösungsansätze für die aktuellen Herausforderungen liegen.

Auch die 47. CIGRE Session 2018 war geprägt von spannenden und fruchtbaren Fachdiskussionen. Einen Auszug daraus finden Sie wie immer in den Ihnen hier vorliegenden Berichten.

Ich wünsche Ihnen eine spannende Lektüre!

Gerhard Christiner

Vorsitzender des Österreichischen Nationalkomitees der CIGRE
Christiner, Gerhard, Austrian Power Grid AG, Wagramer Straße 19, 1220 Wien, Österreich (E-Mail: gerhard.christiner@apg.at) 